\title{
INVESTIGACIONES
}

de HISTORIA ECONÓMICA

2005, primavera, número 2. Pp. 75 a 104

\section{Una larga polémica: el tâtonnement walrasiano}

\section{An endless controversy: the walrasian tâtonnement}

\author{
JULIO SEGURA SÁNCHEZ \\ Universidad Complutense de Madrid
}

\begin{abstract}
RESUMEN
Este trabajo sintetiza la formulación original del tâtonnement para economías de intercambio y producción en las sucesivas ediciones de los Éléments d'économie politique pure y expone y comenta las numerosas reinterpretaciones del mismo desde las primeras críticas de Bertrand y Edgeworth hasta la actualidad. Las conclusiones son: (1) que Léon Walras fue consciente del problema del comercio a precios falsos pero no encontró una formulación adecuada del mismo hasta 1900; (2) que el tâtonnement no pretendía ser una descripción de cómo funcionaban los mercados en la realidad, sino proporcionar un análisis abstracto y teórico de cómo una economía competitiva establecía los precios de equilibrio; (3) que su formulación era estática y (4) que aunque evidentemente no demostró su convergencia, sí señaló el tipo de condiciones que exige la misma.
\end{abstract}

PALABRAS CLAVE: Tâtonnement, Recontratación, Estabilidad, Intercambio a precios falsos

Códigos JEL: B13, B21, B31

\section{ABSTRACT}

This article summarizes the original formulation of Walras's tâtonnement - just for the cases of the theories of exchange and production- as it was first developed in the different editions of the Éléments d'économie politique pure. The article also surveys critically the most relevant reinterpretations from Bertrand and Edgeworth to nowadays. The main conclusions are: (1) Walras was fully aware of the false trade problem since the first edition but he did not find an adequate formulation until the fourth one; (2) Walras's tâtonnement process did not try to describe how markets actually operate but to provide an analytical and abstract tool to deal with the law of the establishment of commodity prices in a competitive economy; (3) the walrasian tâtonnement was on purpose a static device and (4) Walras did not demonstrate tâtonnement' convergence but he pointed toward the kind of conditions needed for it.

KEY WORDS: Tâtonnement, Recontracting, Stability, False trade

JEL Codes: B13, B21, B31 


\section{Introducción ${ }^{1}$}

"Aceptemos el hecho de que la interpretación de la posición de un autor -especialmente si tiene una mente compleja y penetrante- es un problema de inferencia que no puede resolverse mediante la elección de citas"

Stigler (1965), p. 450.

$\mathrm{M}$ arie Esprit Léon Walras (Evreux 1834-Clarens 1910, LW en adelante) ${ }^{2}$ fue el fundador de la teoría del equilibrio general competitivo (en adelante EGC), es decir, del análisis riguroso y formalizado de una economía que asigna los recursos por medio de mercados competitivos y con propiedad privada, y como tal ocupa un lugar tan destacado como Smith, Ricardo o Keynes en la historia del pensamiento económico. En conocidas palabras de Schumpeter, desde el punto de vista de la teoría pura, Walras es el más grande de todos los economistas.

Su interés por la economía fue muy amplio y su propia clasificación de las áreas en que pensaba trabajar distinguía entre la economía pura, la economía política y la economía social, lo que terminó plasmándose, respectivamente, en sus Éléments d'économie politique pure, ou théorie de la richesse sociale (en adelante EEPP) ${ }^{3}$ y en dos colecciones de ensayos (Walras, 1896 y 1898).

$\mathrm{Su}$ trabajo teórico fue el origen de una parte fundamental de los desarrollos del análisis microeconómico que abarcan tres cuartas partes del siglo XX y, por ello, ha pasado a la historia como uno de los principales teóricos de todos los tiempos; pero el resto de su obra no sólo es de elevada calidad, sino que, además, se deriva de su concepción teórica, constituyendo un respetable ejemplo de coherencia, lo que ha sido con frecuencia ignorado, no sólo por la importancia de su planteamiento revolucionario de la teoría del EGC — que habría empequeñecido cualquier otra aportación-, sino también por sus posiciones políticas y sociales, avanzadas para la época y enfrentadas a los dictados de la academia (Segura, 1987, pp. 31-36).

Cuando LW publicó en 1874-77 la primera edición de sus EEPP, fue objeto de una recensión, seis años más tarde, altamente crítica, por parte de Bertrand. La segunda edición de 1889 tuvo más éxito, recibiendo dos comentarios importantes

1 El autor desea agradecer las observaciones realizadas por dos evaluadores anónimos, que han permitido mejorar la presentación y legibilidad del artículo, así como eliminar ciertas expresiones discutibles.

2 Una corta nota autobiográfica de LW puede consultarse en Jaffé (1965), pp. 1-15. En Segura (1987), puede encontrarse un estudio de su vida y obra (pp. 20-55), un cuadro cronológico detallado (pp. 56-69) y su obra completa comentada (pp. 70-100).

3 Walras (1874-77). 
de Edgeworth y Bortkiewicz. Y, en todos ellos, un punto focal fue el proceso de tâtonnement que, en palabras del propio autor, era el procedimiento para comprobar cómo se establecían los precios de equilibrio.

La edición traducida al inglés de los EEPP de Jaffé, en 1954, contenía en sus notas y colación de ediciones numerosas referencias al tema, y la posterior publicación, en tres tomos, de la correspondencia de Walras (Jaffé, 1965) proporcionó muchas pistas sobre la importancia relativa del tâtonnement en el núcleo académico de la época.

En la discusión sobre el tâtonnement walrasiano pueden distinguirse tres etapas:

1. La etapa primera abarca las críticas y comentarios tras la aparición de los EEPP, a las que LW contestó o tuvo en cuenta para introducir modificaciones en subsiguientes ediciones. Incluye los trabajos de Bertrand (1883), Edgeworth (1889 y 1891), Bortkiewicz (1890) y Wicksteed ${ }^{4}$.

2. La segunda etapa corresponde a los trabajos que interpretan o aplican el tâtonnement y que no fueron considerados por LW - ya que la mayoría apareció tras su muerte-, casi todos previos a la salida en 1954 de la edición Jaffé. En ella pueden distinguirse dos enfoques. El primero, referido a las discusiones sobre la naturaleza del tâtonnement, donde las aportaciones esenciales son, entre otras, de Kaldor (1934), Lange (1936) y Schumpeter (1954), que culminan en la síntesis de Patinkin (1956). El segundo atañe a los trabajos pioneros de economía matemática relacionados con la estabilidad del EGC, que incluyen, entre otras muchas, las aportaciones pioneras de Hicks (1939) y Samuelson (1941), sintetizadas por Negishi (1962).

3. La tercera etapa se inicia con un artículo de Jaffé (1967), originado en reacción a los desarrollos del segundo enfoque, a la síntesis de Negishi y al trabajo de Patinkin. A partir de esta publicación, se genera un largo debate cuyos protagonistas principales son Walker (1972, 1987a, 1987b y 1996) y el propio Jaffé (1977 y 1981), que llega hasta nuestros días.

Al día de la fecha, tanto cada una de las etapas como el proceso en su conjunto, tienen algún interés desde distintos puntos de vista.

La primera etapa es, desde la perspectiva de la Historia del Pensamiento Económico, un prototipo de debate entre economistas coetáneos de primera fila sobre un problema analítico con implicaciones prácticas, relativas al funcionamiento de los mercados competitivos. Debate cuyo núcleo es de un genuino interés por avanzar en la clarificación de un tema importante, pero en el que también - como en todos los debates- se deslizan prejuicios, diferencias de escuelas y desacuerdos conceptuales. 
En la segunda etapa, el primer enfoque es el comienzo del ejercicio de una larga búsqueda del sentido del tâtonnement, y un intento de relacionarlo con los desarrollos recientes del análisis económico. El segundo enfoque es una piedra angular de la Historia del Análisis Económico, en la que cristaliza el tema central de la investigación en economía matemática de las siguientes décadas: la existencia, unicidad y estabilidad del EGC.

La tercera etapa es un ejemplo de debate exegético, con un desarrollo muy desigual que incluye, desde puntualizaciones sobre el tratamiento del tâtonnement en LW, hasta interpretaciones extremas, si no inventadas, sobre lo que realmente quiso decir LW en los EEPP e, incluso, intentos de desnaturalizar el enfoque walrasiano.

El objetivo del presente trabajo es doble. Por una parte, descriptivo, resumiendo las posiciones principales de las distintas etapas de la polémica. Por otra parte, interpretativo, dando una opinión personal sobre el sentido del tâtonnement walrasiano en LW, tratando de aplicar la recomendación de Stigler que abre el artículo e intentando que las inferencias sean justificadas.

Esto plantea el problema de cómo interpretar los textos de un autor que escribió hace más de cien años. Existen en nuestro caso dos riesgos.

El primero es analizar la obra a la luz de los instrumentos y desarrollos analíticos disponibles un siglo más tarde, y que se originan, precisamente, en la obra comentada. Con esta perspectiva, es fácil comprobar que LW no demostró la existencia del EGC, ni la unicidad o multiplicidad del mismo, ni menos aún su estabilidad global. Esta comprobación es irrelevante, porque lo sustantivo de la obra de LW es que planteó estos problemas, por vez primera, en un marco del equilibrio general y avanzó soluciones tentativas - formalmente insuficientes- que marcaron un programa de investigación, para docenas de economistas de primera fila, durante un siglo.

Pero el mayor riesgo proviene de la tentación que, para cualquier economista, supone explicar que LW estaba equivocado, y que uno ha descubierto lo que, en caso de ser admitido por los editores, permite además la elaboración de un abundante currículo académico. Y, para ello, nada mejor que destacar los aspectos más oscuros o reelaborados de la obra original e interpretar como 'erróneo', 'ilógico' o 'intento fallido' cualquier argumento complejo que presente alguna inconsistencia indirecta ${ }^{6}$. Trataré de evitar este riesgo con una interpretación constructiva: si existe evidencia de que $L W$ conocía y entendía un problema, resulta, en mi opinión, más adecuado interpretar las omisiones, redacciones abigarradas u oscuras, o insuficiencias de razonamiento como formas inadecuadas o incompletas de expresión de un problema nuevo y difícil, que como errores teóricos sustantivos.

$6 \quad$ En el caso de LW un ejemplo es Bridel y Huck (2002b) y buena parte de la discusión de oral pledges y written pledges de Walker (1987a) y (1996). 
Las aportaciones de este trabajo son limitadas, al tratarse de un autor y de un tema tan estudiados como los que nos ocupan. Por una parte, la literatura existente se articula diferenciando las tres etapas señaladas, las cuales implican enfoques y tratamientos diferentes — tanto temáticos como instrumentales-, que no tiene sentido tratar conjuntamente si se desea tener claro el valor añadido de cada análisis respecto a los precedentes. Por otra parte, se hace un esfuerzo metodológico de interpretación constructiva, en un caso en que la crítica, con frecuencia, se ha centrado en la búsqueda de referencias puntuales sin tener en cuenta el contexto, con el objetivo de perseguir más la detección de errores formales que de señalar la mayor o menor relevancia del tâtonnement en la historia del pensamiento - $-\mathrm{d}$ del análisis- económico. Por último, en las conclusiones se distinguen cuatro temas de distinta naturaleza, que con frecuencia se confunden en el debate sobre el tâtonnement, señalando la articulación entre ellos y la incoherencia que supone defender interpretaciones que requieren argumentos contradictorios, según que se discuta un tema $\mathrm{u}$ otro.

El trabajo se organiza de la siguiente forma: en el epígrafe 2 se presenta el esquema de los EEPP y se describen los tâtonnements ${ }^{7}$ tal y como aparecen formulados en su primera edición; en el epígrafe 3 se sintetiza la polémica original correspondiente a la primera etapa y se señalan las modificaciones introducidas en la explicación de los tâtonnements en subsiguientes ediciones de los EEPP como resultado de algunas críticas; en el epígrafe 4 se presentan las interpretaciones y algunas aplicaciones surgidas en la segunda etapa del debate ${ }^{8}$; en el epígrafe 5 se discuten las interpretaciones alternativas correspondientes a la tercera etapa; $y$, por último, en el epígrafe 6 se expone la interpretación del autor sobre los principales puntos del debate.

Sólo para los modelos de economía de intercambio y de producción, omitiendo el tratamiento en los casos de la formación de capital y la circulación y el dinero, lo que permite no tratar temas complejos de teoría monetaria sin por ello eliminar ningún aspecto relevante de la teoría [véase Kuenne (1961) y Patinkin (1965, nota $C$ del apéndice)]. En palabras de Solow: "Las ecuaciones de intercambio y producción son realmente la cima de los Elements." Solow (1956), p. 88.

8 Puesto que los resultados del enfoque de estabilidad de esta etapa son muy técnicos, sólo se mencionarán en lo que afectan a la interpretación del tâtonnement en los EEPP. El lector interesado en una revisión de la existencia del EGC puede consultar Segura (1970). Para la estabilidad, Negishi (1962). 


\section{El tâtonnement en los EEPP9}

\subsection{El esquema (arquitectónico) de los EEPP}

Para LW, el objetivo de la economía es el estudio de la riqueza social, que es el conjunto de todas las cosas que tienen valor, es decir, que proporcionan utilidad y son escasas (EEPP, Lecciones 3 y 5 ).

Un estudio completo de la riqueza social correctamente definida (EEPP, Lecciones 17 y 18) debe plantearse tres tipos de problemas, que conducen a tres planos de análisis económico (EEPP, Lección 2):

1. La economía política pura, que trata de explicar a qué se debe y cómo se genera la riqueza social. El objetivo de este análisis es la teoría del valor de cambio. Su criterio rector es la veracidad. Constituye un conocimiento de tipo científico.

2. La economía política aplicada, que estudia cómo se produce la riqueza social. El objetivo es la teoría de la industria y su criterio rector la utilidad o el interés. Constituye un conocimiento de tipo práctico, es un arte.

3. La economía social, que estudia cómo se apropia y distribuye la riqueza social. El objetivo es la teoría de la propiedad y su criterio rector la justicia. Pertenece al campo de la ética. Los EEPP corresponden al primer plano de análisis, y su idea central es que todos los componentes de la riqueza social son demandados y ofrecidos en mercados que operan simultáneamente y, por tanto, se influyen entre sí.

El esquema de los EEPP es siempre el mismo. Se selecciona un subconjunto de elementos de la riqueza social partiendo del más sencillo, que se va ampliando sucesivamente ${ }^{10}$, y se procede a:

1. La determinación analítica de las funciones de demanda y oferta de los elementos de la riqueza social objeto de estudio.

2. El análisis de los precios de equilibrio en cuatro fases que, en terminología de LW, son:

1ª La fórmula del equilibrio general o fórmula general de la solución matemática del problema, es decir, la comprobación de que el número de ecuaciones e incógnitas coincide (en el caso de la economía de intercambio, Sección III, Lección 12: 117-124).

9 Las citas textuales a los EEPP aparecerán — salvo que baste citar el epígrafe o lección- como (Lección X: Y, pp. Z), donde X es el número de Lección, Y el del epígrafe y Z la(s) página(s) de la edición Segura (1987), de forma que el lector interesado pueda cotejarlas con los EEPP originales y con la edición Jaffé (1954). Las referencias a la correspondencia de LW se hacen siempre por el número de la carta en Jaffé (1965), incluyendo el volumen y las páginas.

10 Lo que da lugar a cuatro submodelos: la teoría del intercambio puro (EEPP, Secciones II -dos mercancíasy III -varias mercancías); la teoría de la producción (Sección IV); la teoría de la formación de capital (Sección V) y, por último, la teoría monetaria y de la circulación (Sección VI). 
$2^{\mathrm{a}}$ : La ley de establecimiento de los precios de equilibrio, es decir, cómo se alcanzan los precios determinados en el paso anterior (Sección III, Lección 12: 125-130).

$3^{\mathrm{a}}$ : La ley de variación de los precios, es decir, el análisis de estática comparativa (Sección III, Lección 13).

$4^{\mathrm{a}}$ : La combinación de las leyes de variación y establecimiento de los precios de equilibrio, que conduce a la formulación científica de la ley de la oferta y la demanda (Sección III, Lección 13: 138).

El tâtonnement es la pieza central de la segunda etapa del análisis de equilibrio general, el instrumento a través del cual se discute la ley de establecimiento de los precios de equilibrio.

\subsection{El tâtonnement en la primera edición de los EEPP}

Para el caso de la economía de intercambio puro con varios bienes, tras formular el sistema de ecuaciones de igualdad entre oferta y demanda que definen el equilibrio, LW se plantea demostrar que la solución científica del problema del intercambio - la determinación de los precios que igualan las demandas y ofertas de todos los bienes- coincide con la solución empírica que alcanza el mercado competitivo.

Para ello, tras suponer que se vocean al azar en el mercado unos precios de los bienes (B), (C)... en términos de (A) — que es el numerario—, nos encontraremos con que, salvo por casualidad, la oferta y demanda de cada bien no coincidirán. ¿Qué sucederá en los mercados?:

"Si la demanda es superior a la oferta, el precio de dicha mercancía en términos del nume-
rario subirá, si es la oferta la que supera a la demanda, bajará. ¿Qué debemos hacer para
probar que la solución teórica y la solución del mercado son idénticas? Simplemente com-
probar que el alza y la baja [de los precios] son una forma de resolución por tâtonnement del
sistema de igualdades de las ofertas y las demandas." (Lección 12: 125, pp. 298-299).

Tras esto, LW procede a describir el proceso de tâtonnement. Supongamos que los precios voceados al azar de los bienes (B), (C), (D)... son $\mathrm{p}_{\mathrm{b}^{\prime}} \mathrm{p}^{\prime}{ }_{\mathrm{c}^{\prime}} \mathrm{p}^{\prime}{ }_{\mathrm{d}} \ldots$ Como los precios se han determinado aleatoriamente, el valor del exceso de demanda del bien (B) - $\mathrm{F}_{\mathrm{b}}\left(\mathrm{p}_{\mathrm{b}^{\prime}}^{\prime} \mathrm{p}_{\mathrm{c}^{\prime}} \mathrm{p}^{\prime}{ }_{\mathrm{d}} \ldots\right)$ - será, salvo casualidad, no nulo, pero habrá un precio de (B) que anulará su exceso de demanda, es decir, existirá un $\mathrm{p}^{\prime \prime}{ }_{\mathrm{b}}$ que cumpla $\mathrm{F}_{\mathrm{b}}\left(\mathrm{p}^{\prime \prime}{ }_{\mathrm{b}^{\prime}} \mathrm{p}^{\prime}{ }_{\mathrm{c}^{\prime}}\right.$ $\left.\mathrm{p}^{\prime}{ }_{\mathrm{d}} \ldots\right)=0$. El siguiente paso es, manteniendo $\mathrm{p}^{\prime \prime}{ }_{\mathrm{b}^{\prime}}$ buscar el valor del precio de $(\mathrm{C})$, $\mathrm{p}^{\prime \prime}{ }_{\mathrm{c}}$ que anula $\mathrm{F}_{\mathrm{c}}\left(\mathrm{p}^{\prime \prime}{ }_{\mathrm{b}^{\prime}} \mathrm{p}^{\prime \prime}{ }_{\mathrm{c}^{\prime}} \mathrm{p}_{\mathrm{d}^{\prime}}, \ldots\right)$ y así sucesivamente (Lección 12: 129).

Cuando se obtiene $\mathrm{p}^{\prime \prime}{ }_{\mathrm{c}^{\prime}}$ resultará que $\mathrm{F}_{\mathrm{b}}\left(\mathrm{p}^{\prime \prime}{ }_{\mathrm{b}^{\prime}}, \mathrm{p}^{\prime \prime}{ }_{\mathrm{c}^{\prime}} \mathrm{p}^{\prime}{ }_{\mathrm{d}} \ldots\right)$ ya no será nula, pero LW argumenta que se encontrará más cerca de 0 que $\mathrm{F}_{\mathrm{b}}\left(\mathrm{p}_{\mathrm{b}^{\prime}} \mathrm{p}_{\mathrm{c}^{\prime}} \mathrm{p}^{\prime}{ }_{\mathrm{d}} \ldots\right)$, con lo que el proceso descrito será convergente. Puesto que éste es un punto esencial del debate, conviene conocer textualmente el argumento de LW: 
“Esto [la convergencia] parece probable si se recuerda que el cambio de $\mathrm{p}_{\mathrm{b}}{ }_{\mathrm{b}}$ a $\mathrm{p}_{\mathrm{b}}$ que convirtió esta última desigualdad $\left[\mathrm{F}_{\mathrm{b}}\left(\mathrm{p}_{\mathrm{b}^{\prime}}^{\prime} \mathrm{p}_{\mathrm{c}^{\prime}}^{\prime} \mathrm{p}_{\mathrm{d}}^{\prime} \ldots\right) \neq 0\right]$ en igualdad, tiene efectos directos $y$, al menos en lo que concierne a la demanda de (B) todos del mismo sentido [hacia la igualdad], mientras que los cambios de $\mathrm{p}_{\mathrm{c}}^{\prime}$ a $\mathrm{p}^{\prime \prime}{ }_{\mathrm{c}^{\prime}}$ de $\mathrm{p}_{\mathrm{d}}{ }_{\mathrm{d}}$ a $\mathrm{p}_{\mathrm{d}}{ }^{\prime}$.. que alejaban de la igualdad la desigualdad precedente, tienen efectos indirectos $\mathrm{y}$, al menos en lo que concierne a la demanda de (B), de sentido contrario, compensándose hasta cierto punto los unos con los otros. Por este motivo el sistema de nuevos precios $\mathrm{p}^{\prime \prime} \mathrm{b}^{\prime} \mathrm{p}^{\prime \prime}{ }_{\mathrm{c}^{\prime}}$ $\mathrm{p}^{\prime \prime}{ }_{\mathrm{d}}$... es más cercano al equilibrio que el sistema de viejos precios $\mathrm{p}_{\mathrm{b}^{\prime}}^{\prime} \mathrm{p}_{\mathrm{c}^{\prime}}^{\prime} \mathrm{p}_{\mathrm{d}}^{\prime} \ldots$ y no hay más que seguir el mismo método para acercarse más y más al mismo." (Lección 12: 130, p. 302).

Para el caso de una economía de producción, el tâtonnement juega la misma función, pero es doble, porque es preciso ajustar dos tipos de mercados: los de productos y los de servicios productivos.

El primer tâtonnement se desarrolla en los mercados de productos: suponiendo constante la demanda de cada producto, se ajustan las cantidades ofrecidas no los precios- hasta el punto en que el precio de venta se iguala al coste medio de producción para cada bien ${ }^{11}$.

El segundo tâtonnement se desarrolla en los mercados de servicios productivos: suponiendo constante la oferta de cada tipo de servicio productivo, se ajustan los precios de los mismos hasta el punto en que se igualan las demandas y ofertas.

\section{La primera etapa: la polémica con Walras}

\subsection{Bertrand}

En un famoso artículo, Bertrand (1883) ${ }^{12}$ realiza una crítica de la primera edición de los EEPP, en la que señala un problema que será fundamental en todos los debates posteriores sobre el tâtonnement: el intercambio a precios de no equilibrio, también llamado intercambio a precios falsos. La idea es simple: a lo largo del proceso se van

11 Esto implica que el beneficio del empresario es nulo. El papel del empresario para LW es el de conector de los mercados de factores y productos, tal y como detectó con perspicacia Edgeworth (1925, vol. II, pp. 309 y ss.). Sin embargo, Edgeworth (1889) criticó duramente el supuesto de beneficios nulos al no comprender cabalmente la razón para ello: que el empresario walrasiano es un mero combinador de servicios productivos arrendados a los capitalistas, trabajadores y terratenientes cuya retribución está incluida en los costes de producción. Véase, por ejemplo, EEPP, Lección 18: 188, p. 386; y Lección 19; 199, p. 398.

12 Más famoso por la crítica a la obra de Cournot, ya que se propone una forma de competencia a corto plazo en el duopolio alternativa, que dio lugar al llamado modelo de Bertrand. 
voceando unos precios para los que los excesos de demanda de los bienes son no nulos, es decir, unos precios de no equilibrio. Si cuando se vocea al azar el primer vector de precios se realizan intercambios, que es la única forma de constatar que los mercados no se vacían, cuando se vocee el nuevo vector de precios, las cantidades poseídas de los bienes por los individuos serán distintas que las dotaciones iniciales y lo será también su riqueza (renta). Aunque el proceso sea convergente y terminen alcanzándose unos precios que vacíen todos los mercados, no serán los que solucionan el sistema de ecuaciones de intercambio, especificadas para unas dotaciones iniciales dadas, porque éstas han ido cambiando en cada intercambio a precios falsos.

Esto implica que no es correcta la afirmación de LW de que la solución científica del problema del intercambio es 'la misma' que la que proporciona el mercado por el mecanismo de la competencia ${ }^{13}$. Puesto que, en opinión de Bertrand, en los mercados reales se realizan intercambios a precios falsos, tanto el equilibrio empírico, como el resultante del tâtonnement, son indeterminados.

La crítica es, sin lugar a dudas, correcta, y que LW pensó sobre ella, pese a no mantener relación escrita alguna con Bertrand, se comprueba tanto en su correspondencia $^{14}$ como en el hecho de que, en la segunda edición de los EEPP (1889), añadiera en el epígrafe 101 (102 de la edición definitiva):

“El teórico tiene derecho a suponer que los elementos determinantes de los precios son constantes en el período de tiempo que ha elegido para formular la ley del establecimiento de los precios de equilibrio [...] por lo demás, la primera formulación [ley de establecimiento] conduce inmediatamente a la segunda [ley de variación]. En efecto, los factores que se encuentran tras el establecimiento de los precios son también los que se encuentran tras la variación de los mismos." (Lección 10: 102, p. 250).

Si se interpreta que la crítica de Bertrand se refería al realismo del tâtonnement, el añadido de LW no la contesta, pero aporta evidencia de que LW consideraba que, a lo largo del proceso, los "elementos determinantes" del equilibrio permanecían constantes y esos elementos son las utilidades de los bienes y las cantidades poseídas de las mercancías por cada individuo. Volveremos a encontrarnos con este problema.

Véase EEPP, Lección 12: 124, p. 298.

En carta a Haton de la Goupillière, profesor de la École des Mines (nº 396, vol. I, pp. 552-553), de fecha tan temprana como finales de 1877, LW da cuenta de un breve encuentro, en una estación, con Bertrand, en el que se manifestaron las diferencias en el tema que nos ocupa. 


\subsection{Edgeworth y Bortkiewicz}

La crítica de Edgeworth (1889) ${ }^{15}$ a la segunda edición de los EEPP es más amplia, pero en lo relativo al tâtonnement puede sintetizarse en dos puntos ${ }^{16}$ :

1. Confundir el sentido de las ecuaciones de equilibrio, que son estáticas y, por tanto, sólo pueden definir el equilibrio, con un proceso dinámico que trata de explicar la senda que se sigue para alcanzar el equilibrio.

2. Definir una senda, no la senda hacia el equilibrio, considerando que si se trata de tipificar el comportamiento económico que subyace a la senda dinámica de ajuste (no de definirla), la teoría de la recontratación, formulada por el propio Edgeworth ${ }^{17}$, constituye una explicación más satisfactoria que el tâtonnement walrasiano. Dicha senda, además, depende de la estructura de mercado.

Ambas críticas parten de la base de que Edgeworth consideraba la teoría de LW como una descripción estilizada de la tendencia real que los precios seguían hacia el equilibrio, y una descripción de carácter dinámico que trataba de identificar la verdadera senda seguida por los precios.

Respecto al primer punto, Bortkiewicz (1890) señaló acertadamente que LW no consideró en ningún lugar los tâtonnements como una secuencia dinámica de hechos de mercado, ya que la característica básica del análisis estático es la consideración de que los individuos poseen cantidades fijas, y eso es lo que supone LW (cantidades fijas de mercancías en intercambio, de servicios productivos en producción). Además, criticó con agudeza la contradicción entre esta crítica a LW y el aprecio de Edgeworth por el trabajo de Cournot, que utilizaba una variante de tâtonnement en su tratamiento de la competencia entre duopolistas.

Pero, además, existe evidencia de que el propio LW tenía claro el carácter estático de las ecuaciones del sistema que define el equilibrio ${ }^{18}$ y que, por lo tanto, distinguía entre éstas y los aspectos supuestamente dinámicos de las variaciones de los precios que conducen a la consecución del equilibrio. La segunda crítica considera que el tâtonnement no es la forma idónea de modelar el proceso de ajuste de los precios y se relaciona, de nuevo, con el problema del intercambio a precios falsos.

Edgeworth coincidía con Bertrand en que, para obtener los precios de equilibrio mediante un proceso de prueba y error, era preciso que no se realizaran intercambios efectivos, en etapas previas, a precios distintos de los de equilibrio. Él mismo había propuesto un mecanismo para obviar este problema, la recontratación: si se supone

\footnotetext{
15 Completada dos años más tarde en Edgeworth (1891) y, con una nota añadida, en Edgeworth (1925), p. 311.

16 Una descripción completa de las críticas de Edgeworth puede verse en Walker (1987b).

17 Véase Edgeworth (1881), p. 17.

18 Jaffé (1965), carta nº 1.200 a Pareto, 9-1-1895, vol. II, Anexo, p. 630.
} 
que, tanto los compradores como los vendedores, pueden recontratar las cantidades que hayan intercambiado a cualesquiera precios, suscribirán contratos provisionales hasta que los intercambios se realicen a unos precios para los que nadie obtenga ventaja alguna de recontratar, en cuyo momento los contratos se harán efectivos. Como es claro, sólo a precios de equilibrio ni compradores ni vendedores obtienen ventaja alguna de recontratar.

Resulta inevitable pensar que la crítica de Edgeworth tiene que ver, más con la defensa de su recontratación como mecanismo para evitar el intercambio a precios falsos, que con los defectos del tâtonnement en sí mismo, como lo demuestra el juicio de intenciones sobre LW, añadido en 1925 a su trabajo de 1891, según el cual el proceso de voceo de precios tenía como objetivo 'distraer la atención' sobre un proceso más fundamental, que era su recontratación ${ }^{19}$.

Que LW describía una senda y no la senda, es una crítica correcta pero muy débil. Lo cierto es que LW nunca mencionó senda alguna, por lo que el tâtonnement no es ni mejor ni peor que el recontracting, sino algo distinto ${ }^{20}$.

La opinión de que la senda seguida por los precios depende de la estructura de mercado es cierta, y se encuentra profusamente ilustrada por Edgeworth en su referencia a que existen mercados en que los precios se determinan mediante subasta inglesa, en otros por la holandesa; en unos mercados los distintos precios de los bienes son simultáneos, en otros sucesivos; en otros mercados, incluso, hay precios distintos para comprador y vendedor. Pero es difícil pensar que esto sea una crítica a LW, que siempre consideró que la estructura de mercados era competitiva.

Pero, sobre todo, para Edgeworth el tema de la senda no era científico, por lo que se tratase de una o de la debería haber sido poco relevante ${ }^{21}$.

\subsection{Wicksteed}

Wicksteed señaló a LW una dificultad en su argumentación de que el proceso del tâtonnement terminaba alcanzando los precios de equilibrio. Aunque el comentario se refiere al segundo tâtonnement de la teoría de la producción, el que se realiza sobre los precios de los servicios (Lección 21: 217), es válido con carácter general. Dados los tres servicios productivos, tierra $(\mathrm{T})$, trabajo $(\mathrm{P})$ y capital $(\mathrm{K})$, Wicksteed señalaba

Véase Edgeworth (1925), vol. II, p. 311.

Véase Jaffé (1981) y Walker (1987b).

"El toma y daca del intercambio por medio del cual se determina el precio, la dirección que el sistema sigue para llegar a la posición de equilibrio, no es una parte del dominio de la ciencia." (Edgeworth, 1891, p. 12). 
que los cambios en sentido contrario de $\mathrm{p}_{\mathrm{p}} \mathrm{y} \mathrm{p}_{\mathrm{k}}$ podían tener un efecto acumulativo sobre la oferta de (T) y contrario al efecto directo del cambio en $\mathrm{p}_{\mathrm{t}^{\prime}}$ e incluso que los cambios en $p_{p}$ podían no sólo contrarrestar los efectos del cambio en $p_{k}$ sino, también, en $p_{t}$. Como es claro, en este caso, el proceso no encontraría los precios de equilibrio, aunque Wicksteed creyera que era poco probable ${ }^{22}$.

Wicksteed no lo demostró, no porque según su modesta opinión sus matemáticas fueran débiles, sino porque no es posible demostrarlo más que bajo supuestos muy específicos, como se comprobaría siete décadas más tarde ${ }^{23}$.

La respuesta de LW pocos días más tarde agradecía, pero minimizaba, la observación de Wicksteed, señalando que:

"En cuanto a la demanda [de T], el aumento de [del precio de] (P) y la baja de [del de] $(\mathrm{K})$ en que uno tiende a hacer más caro y otro más barato el producto, tienen efectos que se compensan hasta cierto punto; en cuanto a la oferta [de T] los efectos pueden acumularse pero, como son indirectos y no directos, es probable que generalmente la igualdad entre la oferta y la demanda de (T) no se vea sensiblemente alterada" ${ }^{24}$.

Como es obvio, LW no contesta más allá de lo que aparece en la primera edición de los EEPP, pero que tuvo en cuenta la observación es claro si se constata que incluyó, en la segunda (1889) y sucesivas ediciones de los EEPP en el epígrafe 213 (217 de la edición definitiva), la matización de "al menos en lo que concierne a la demanda de $(\mathrm{T})$ ", tanto al hablar del efecto directo del cambio de $\mathrm{p}_{\mathrm{t}^{\prime}}$ como del indirecto del cambio en $\mathrm{p}_{\mathrm{p}} \mathrm{y} \mathrm{p}_{\mathrm{k}}$.

Wicksteed insistió en el tema cuando revisó la teoría del intercambio de la segunda edición de los EEPP. En una larga carta a LW, en la que hacía numerosas observaciones técnicas, sugirió una nueva redacción del epígrafe 128 (130 de la edición definitiva), que implicaba un cambio en la interpretación de la convergencia del tâtonnement, dándole un contenido dinámico que hasta entonces no había manifestado. La sugerencia era que cuando se analizaba, por ejemplo, el exceso de demanda de (B), las variaciones de $\mathrm{p}_{\mathrm{b}}$ debían, y las de $\mathrm{p}_{\mathrm{c}^{\prime}} \mathrm{p}_{\mathrm{d}}$... podian, hacerla tender hacia la igualdad y que en caso de que estas últimas tendieran a producir la igualdad (mayor desigualdad) del exceso de demanda de (B), las variaciones de $\mathrm{p}_{\mathrm{b}}$ no necesitaban ser tan (debían ser más) rápidas ${ }^{25}$.

La observación implica una concepción en que la velocidad de reacción de los precios resulta relevante para la convergencia del proceso y, además, debe ser distinta

\footnotetext{
Véase carta n ${ }^{\circ} 619$ de 1-12-1884, vol. II, p. 17.

Véase nota 28.

Carta $n^{\circ} 627,14-12-1984$, vol. II, p. 25.

Carta n $^{\circ}$ 875, 2-4-1889, vol. II, Anexo, p. 303.
} 
para unos bienes y otros, lo que requeriría hipótesis adicionales poco plausibles sobre el proceso de voceo de los precios. Esta observación no fue contestada ni afectó a versiones futuras de los EEPP26.

\subsection{El resultado de la polémica: los bons}

Aparte de la frase añadida por dos veces en la segunda edición, ya mencionada, y de una mejora formal de redacción introducida en dicha edición, en el nuevo epígrafe 203 de la Lección 20 (207 de la edición definitiva), existe una modificación sustancial del tâtonnement en la cuarta edición.

En la primera edición de los EEPP, LW había señalado, al plantear el tâtonnement en la teoría de la producción, que existía una complicación adicional al caso del intercambio. En este último, las cantidades totales de bienes no se modificaban, pero la producción se caracteriza por transformar servicios productivos en productos, por lo que, al vocear unos precios de aquéllos, se fabricarían determinadas cantidades de productos y, por tanto, al vocearse nuevos precios sería preciso fabricar nuevas cantidades de productos. Como esto implicaría un problema similar al de comercio a precios falsos señalado por Bertrand y Edgeworth, LW añadió:

"Para formular un tâtonnement riguroso en la producción, tal y como hicimos para el intercambio, teniendo en cuenta esta circunstancia, no hay mas que suponer que los empresarios representan por medio de vales [sur 'bons'] las sucesivas cantidades de productos que primero determinan al azar y después aumentan o disminuyen respectivamente según que el precio de venta exceda al coste de producción o viceversa hasta que se igualen ambos; y que los terratenientes, trabajadores y capitalistas representen de igual forma mediante vales las sucesivas cantidades de servicios que primero determinan al azar y después aumentan o disminuyen respectivamente según que la demanda exceda a la oferta o al revés hasta que se igualen ambas." (Lección 20: 207, p. 406).

Los bons walrasianos aparecen como contratos contingentes, que sólo se ejecutarán cuando se hayan alcanzado los precios de equilibrio y, por tanto, estén respaldados por las producciones y cantidades ofrecidas de servicios de equilibrio. Que para LW los contratos no se realizaban hasta que se lograba el equilibrio está fuera de toda duda:

26 De hecho, la correspondencia técnica entre ambos autores termina un mes más tarde, el 3 de mayo, fecha en la que LW contesta a la extensa carta de observaciones citada en el texto con un breve comentario sobre el final degree of utility de Jevons. 
"Por tanto el equilibrio de la producción se establecerá en primer lugar en principio. Se establecerá después efectivamente por medio de la entrega recíproca de los servicios a obtener y los productos a fabricar..." (Lección 20: 207, p. 406).

Pero LW señala una dificultad adicional: el tiempo. Mientras que al vocearse los precios de equilibrio los intercambios pueden realizarse de forma inmediata, porque ya existen las cantidades dadas inicialmente de bienes, en la producción, al vocearse los precios de equilibrio de bienes y servicios productivos, la venta de los segundos a los empresarios puede ser inmediata, pero la producción exige un cierto plazo. La solución de esta segunda dificultad es obvia "pura y simplemente haciendo abstracción del plazo", completando la cita anterior con:

“[...] a lo largo de un cierto período de tiempo durante el cual los datos del problema no cambian." (Lección 20: 207, p. 406).

No parece, por tanto, haber duda alguna de que los bons walrasianos están diseñados para evitar las transacciones a precios falsos en su modelo de producción. Sin embargo, en la medida en que no introdujo el mismo mecanismo en el tâtonnement del intercambio, la crítica posterior ha seguido discutiendo si 'realmente' LW eliminó este problema en su modelo de intercambio. Un tema que volveremos a encontrarnos.

\section{La segunda etapa}

Las discusiones sobre el tâtonnement generadas tras la muerte de LW en 1910, y previas al artículo de Jaffé (1967), pueden clasificarse en dos grupos. El primero incluye las interpretaciones generales que tratan fundamentalmente dos problemas - el intercambio a precios falsos y el carácter dinámico o no del proceso-, y el segundo inserta el tâtonnement explícitamente en el tema de la estabilidad del equilibrio. Los trataré por separado.

\subsection{De Kaldor a Patinkin: la naturaleza del tâtonnement}

En un artículo poco citado en relación a su importancia, Kaldor (1934), discutiendo la condición de que todos los intercambios deben realizarse a los mismos precios, señala que esto puede lograrse por dos vías, describiendo la primera como sigue: 
“Los compradores y vendedores, coincidiendo simultáneamente en el mercado, 'voceen' precios, revisen y vuelvan a revisar sus ofertas, pero no realicen ningún intercambio hasta que alcancen un sistema de precios que asegure el equilibrio para cada uno y en todas las partes del mercado (supuesto de Walras)" (Kaldor, 1934, p. 126).

En la interpretación "kaldoriana" no hay duda de que LW excluye el intercambio a precios falsos. El supuesto de voceo de precios cumple una función clara: en el mercado real, los precios no se determinan por un procedimiento de prueba y error, y los tanteos se hacen con contratos provisionales no irreversibles, porque la formación del precio de equilibrio no es un resultado del proceso de intercambio, sino que debe precederlo (Kaldor, 1934, p. 127).

Además, el 'voceo' de precios es un método para lograr que los precios finales sean únicos, sin relación alguna con procesos dinámicos, estabilidad, convergencia, sendas, realismo o temas afines. Se trata de la interpretación más restringida del tâtonnement cuyo mérito radica, por ello, en destacar lo sustancial del mecanismo: que es un instrumento para obtener precios únicos de equilibrio.

Lange (1936), discutiendo la viabilidad del socialismo en el sentido de cálculo económico capaz de computar en una oficina central unos precios como los del mercado, propuso un procedimiento iterativo de intercambio de información entre un centro planificador y los agentes privados en el que aquél hacía de subastador. En la medida en que el centro planificador no permitía realizar intercambios hasta que comunicara a los agentes individuales los precios que casaban ofertas y demandas, es claro que se trataba de un tâtonnement sin intercambio a precios falsos. Pese a ello, Lange vincula directamente el proceso walrasiano con el análisis dinámico, sumándose así a la visión dinámica del tâtonnement iniciada por Edgeworth:

“El método tradicional de tratar la estabilidad del equilibrio, tal y como lo aplicaron Walras, Marshall y Hicks, es una forma implícita (y por tanto imperfecta) de análisis dinámico" (Lange, 1944, p. 94).

Pero en el ámbito de la Historia del Pensamiento Económico fue posiblemente Schumpeter, por la influencia de su History, quien más contribuyó a cristalizar la visión dinámica del tâtonnement. Tras defender a LW de algunas críticas sobre las insuficiencias matemáticas de su teoría, señaló:

\footnotetext{
“De todos modos, es verdad que trató el problema de la estabilidad de un modo muy peculiar, porque lo puso en relación con algo que desde el punto de vista lógico es un problema por completo diferente, a saber, el problema de la relación entre la solución matemática de sus ecuaciones y los procesos de un mercado real cualquiera." (Schumpeter, 1971, p. 1.096).
} 
Es correcto señalar la identificación formal de los dos primeros problemas en los EEPP provocada, como el propio Schumpeter señala, por la obsesión de LW en convencer de que la solución empírica - la del mercado competitivo- y la científica la solución del sistema de ecuaciones - proporcionaban la misma configuración de equilibrio. Pero es cuanto mínimo dudoso que LW se planteara el tâtonnement como un problema de estabilidad (dinámica global).

El trabajo más ambicioso y completo de este período es el de Patinkin (1956). En la nota (B) del apéndice, titulada "Walras's Theory of Tâtonnement", además de revisar prácticamente todas las aportaciones a la polémica desde Edgeworth hasta las notas de la edición Jaffé de 1954, Patinkin sostiene posiciones claras sobre tres puntos fundamentales.

El primero, el comercio a precios falsos. Para Patinkin, LW no fue consciente de la necesidad lógica de la recontratación en la economía de intercambio porque las cantidades de bienes estaban dadas, y no se percató de que en una economía de producción los datos son las cantidades de servicios productivos y no las de bienes como en una economía de intercambio (Patinkin, 1965, p. 534).

La opinión parece equivocada. La referencia de Patinkin (EEPP, Lección 20, p. 238, línea 4) más bien dice lo contrario, ya que las líneas 3 y 4 rezan:

"Dicho esto, sea un individuo que posee $\mathrm{q}_{t}$ de $(\mathrm{T}), \mathrm{q}_{\mathrm{p}}$ de $(\mathrm{P}), \mathrm{q}_{\mathrm{k}}$ de $(\mathrm{K}) \ldots$... (EEPP, edición definitiva, p. 209).

Es decir, las dotaciones iniciales del individuo son las cantidades de factores: tierra $(\mathrm{T})$, trabajo $(\mathrm{P})$ y capital $(\mathrm{K})$.

El segundo tema es el relativo al realismo o no del tâtonnement. Sobre esto la interpretación es, en mi opinión, definitiva:

"Walras estaba interesado fundamentalmente en describir no la senda real hacia el equilibrio, sino la naturaleza de las fuerzas automáticas que impulsan a la economía a lo largo de la senda, fuera ésta la que fuera." (Patinkin, 1965, p. 537).

En opinión de Patinkin, LW no trataba de explicar hechos de mercado como creía Edgeworth, ni estaba interesado por la senda.

El tercer tema es el de la relación con la estabilidad del proceso. Patinkin, tras la interpretación comentada sobre el comercio a precios falsos, analiza el tâtonnement en caso de que existiera recontratación. Señala con acierto que el razonamiento de LW no es suficiente ${ }^{27}$, porque la simple mención literaria a la menor importancia de los 
efectos indirectos no constituye una demostración, pero separa nítidamente el problema que trata de discutir el tâtonnement del problema de estabilidad del equilibrio, señalando que LW discute en los EEPP la estabilidad antes de formular el tâtonnement y que, después, en las numerosas veces que describe el proceso, nunca utiliza el término 'estabilidad', ni relaciona el proceso con su análisis previo. Y, a mayor abundamiento, al tratar las aportaciones de los economistas matemáticos -básicamente Wald-critica sus interpretaciones en relación al tâtonnement porque ignoran que, en el proceso walrasiano, hay algo más fundamental que la existencia o la unicidad del equilibrio: “cómo el mercado alcanza esa solución” (Patinkin, 1965, p. 538).

\subsection{El tâtonnement como análisis de estabilidad}

Desde la década de 1930, la teoría del equilibrio general comenzó a desarrollarse por métodos matemáticos avanzados. El primer problema abordado, el de existencia del equilibrio, fue inicialmente resuelto de forma rigurosa en los trabajos de Wald (193335); pero es el segundo problema, el de la estabilidad, el que resulta relevante en este contexto.

En su revisión sobre la estabilidad de una economía competitiva, Negishi (1962) consideró el tâtonnement walrasiano como un tipo de análisis de la dinámica del proceso de vaciamiento de los mercados. Negishi consideró que el análisis de LW era correcto para el caso de intercambio de dos bienes, pero que falló en la demostración para el caso de varios bienes, tarea que culminó con éxito Hicks en 1939, al formular sus condiciones de estabilidad estática perfecta. Para Negishi, el análisis de LW -y el de Hicks - era de carácter estático, como puso de manifiesto Samuelson (1941), ya que no incluía una regla de comportamiento dinámico de los precios. No obstante, existen relaciones significativas entre la estabilidad estática, útil para los ejercicios de estática comparativa, y el análisis propiamente dinámico de la estabilidad del equilibrio competitivo.

No es preciso leer el trabajo comentado para intuir que su interés no se centra, ni en la interpretación de Walras, ni en la naturaleza del tâtonnement, y que éste se utiliza como categoría genérica. Como es obvio, el artículo se centra en las condiciones conocidas en la época de estabilidad local y global ${ }^{28}$, pero Negishi analiza dos tipos de procesos dinámicos y los bautiza: los procesos de tâtonnement son aquellos en los que la variación del precio de un bien tiene el mismo signo que el del exceso de demanda de ese bien y existe recontratación, constituyendo una "versión 
simplificada de la moderna formulación del tâtonnement walrasiano", mientras que los procesos de no-tâtonnement son aquellos en que tiene lugar intercambio a precios falsos.

En resumen, y en lo que aquí concierne, el trabajo de Negishi sintetiza la posición dominante -y desde la perspectiva de análisis de estabilidad, correcta- de la literatura: el tâtonnement es un instrumento de naturaleza estática que trata de describir estilizadamente cómo funcionan los mercados competitivos organizados y en el que no existe comercio a precios de no equilibrio.

\section{La tercera etapa: reinterpretaciones del tâtonnement, Jaffé y Walker}

En un trabajo seminal Jaffé (1967) se plantea:

"recordar y reevaluar una vez más [en referencia a Jaffé (1954) y (1965)] la teoría del tâtonnement de Léon Walras, tal y como fue inicialmente expuesta en los Élements d'Économie Politique Pure." (Jaffé, 1967, p. 1).

La motivación es clara: las "sutilezas del análisis moderno" (es decir, el enfoque descrito en 4.2.) habían desvirtuado el tâtonnement de LW y guiado la interpretación del mismo en las décadas precedentes en una dirección inadecuada. Pese a que el trabajo comentado contiene muchas ramificaciones, sintetizaré la posición de Jaffé en los tres puntos que considero implican aportaciones relevantes —sean o no correctas en mi opinión ${ }^{29}$-: el problema del intercambio a precios falsos, la corrección del tratamiento del tâtonnement y su carácter realista o abstracto ${ }^{30}$.

Respecto al intercambio a precios falsos, la posición de Jaffé es nítida: LW no lo tuvo en cuenta, no en el sentido de excluirlo, sino en el de no percatarse de sus efectos, y por eso falló en su intento de demostrar que la solución teórica y la de mercado eran idénticas (EEPP, Lección 12: 125) ${ }^{31}$. Aunque admitió el intercambio a precios falsos en la descripción del mercado de deuda francesa al 3\% (rentes, EEPP, Lección 5: 42), omitió el mismo en la discusión básica del tâtonnement (EEPP, Lección 12: 125130). En este punto, la aportación crucial de Jaffé es destacar por primera vez que el

29 Y, en algunos puntos, en la del propio autor que, como veremos más adelante, en Jaffé (1981) modificó substancialmente ciertas interpretaciones de 1967.

30 Omito, por razones de espacio, las críticas a algunas interpretaciones de economistas matemáticos como Goodwin, Metzler, Newman, Negishi o Uzawa.

31 En palabras de Jaffé: “Es el mecanismo impersonal de la competencia pura en un mercado perfecto [...] lo que proporciona, tal y como Walras lo veía, la misma solución que sólo podría obtener algebraicamente un intellectus angelicus tipo ordenador que conociera todos los parámetros" Jaffé (1967), p. 2. 
teorema de las redistribuciones equivalentes ${ }^{32}$ implicaba para LW que una condición como mínimo suficiente para que el mercado tuviera una solución única era que el valor de las dotaciones iniciales de los individuos fuera constante a lo largo de todo el proceso, lo que no sucedería en caso de que hubiera intercambio a precios de no equilibrio. Y, pese a la introducción de los bons en la edición definitiva de los EEPP, Jaffé acepta la interpretación de Patinkin (1965) de que la no consideración de bons o algún mecanismo de recontratación en su teoría del intercambio, implica que LW no fue consciente de los efectos del intercambio a precios falsos.

No menos contundente es la opinión de Jaffé sobre la corrección del proceso, señalando que el planteamiento de LW fue "totalmente inadecuado", quedando muy alejado del ideal proclamado de que las demostraciones debían ser rigurosas, siendo el argumento esencial que LW consideraba "probable" la convergencia del proceso $^{33}$, lo que no constituye una demostración y, además, que suponía implícitamente que dicha convergencia era hacia el equilibrio definido por su sistema de ecuaciones, lo que no era posible, ya que no había tenido en consideración los efectos distorsionantes de los intercambios a precios falsos.

Por último, en lo relativo al carácter realista o abstracto del tâtonnement, la opinión de Jaffé es que la raíz del mismo era introducir un "aire de relevancia empírica" en el modelo abstracto de EGC, por lo que la introducción de bons en la cuarta edición de los EEPP, lejos de poder considerarse un paso adicional en la idealización del verdadero sistema de mercado:

"Es, de hecho, un abandono del realismo, y con dicho abandono se pierde de vista el propósito inicial de la teoría del tâtonnement." (Jaffé, 1965, p. 12).

El segundo trabajo fundamental en el debate de esta etapa es el de Walker (1972). Partiendo de una interpretación empirista, Walker señala que un tâtonnement debe tener ciertas propiedades: un único precio en cada momento para que pueda existir un valor de los excesos de demanda, un procedimiento de difusión de la información entre los participantes, un procedimiento para calcular los excesos de demanda a cada vector de precios y la ausencia de comercio a precios falsos. En opinión de Walker, tales propiedades no las proporcionan las fuerzas competitivas impersonales, sino las instituciones y las reglas del mercado y:

“Dadas varias mercancías en un mercado en estado de equilibrio general, los precios corrientes de estas mercancías no cambiarán si se redistribuyen las cantidades de éstas entre los individuos de una forma cualquiera, siempre que la suma de las cantidades poseídas por cada uno de los individuos se mantenga igual en valor." (EEPP, Lección 14: 143, p. 318). 
“Walras desarrolló un modelo [...] en el que no proporcionó marco específico estructural alguno para generarlo. Dio la impresión de que en un mercado competitivo puro el proceso equilibrador operaba por sí mismo por la acción de fuerzas impersonales." (Walker, 1972, p. 346).

La crítica al tâtonnement de LW se centra, por tanto, en la ausencia de marco institucional y procedimientos operativos: ¿quién anuncia los precios?, ¿cuál es el procedimiento (no la regla) para cambiar los precios?, ¿cómo se justifica que no haya intercambio a precios falsos? y ¿cuál es el procedimiento de recogida y difusión de la información? Esta crítica es generalizable a todos los autores que utilizan tâtonnements de tipo walrasiano ${ }^{34}$ y resulta especialmente relevante porque en ellos se basa el moderno tratamiento de la estabilidad.

El proceso walrasiano no es para Walker un modelo de tâtonnement competitivo completo: en los modelos en que existe un "fijador de precios omnisciente" (à la Edgeworth), porque nunca seguirá la regla del exceso de demanda walrasiana y resultan irrelevantes los temas de estabilidad; en los modelos con "fijador de precios informado", porque faltan los requisitos institucionales y las reglas de funcionamiento e, incluso, falla el análisis de estabilidad ${ }^{35}$.

Llevando al extremo esta visión empirista ${ }^{36}$, en una sucesión de trabajos reformulados en Walker (1996), el autor llega a sostener que las ediciones segunda y tercera de los EEPP — que no contienen bons - tienen una categoría teórica superior a la cuarta definitiva porque:

"Walras no diseñó el proceso de tâtonnement de precios o de producción como un esquema utópico sino, pensaba, como una descripción de los ajustes reales." (Walker, 1996, p. 51).

En su trabajo póstumo, Jaffé (1981) modifica de forma radical la posición sostenida en 1967. Si su reinterpretación se hubiera limitado a dudar del "aire de relevancia empírica" del tâtonnement defendido entonces, concluyendo que el proceso walrasiano perseguía "[...] describir una posibilidad empírica o un desideratum factible mas que un hecho empírico" (Jaffé, 1981, p. 315), poco habría que oponer ${ }^{37}$, pero el cambio de posición es mucho más amplio y discutible.

El origen de la nueva posición es un trabajo previo (Jaffé, 1977) en el que considera que los EEPP tienen un claro sesgo normativo, apoyándose en la discusión de

\footnotetext{
$34 \quad$ Lange (1936) y Wicksell (1934) entre otros.

35 Porque al fijador de precios "su experiencia no le dice nada; no tiene directrices, no persigue objetivo alguno, no sigue otras reglas de precios que las walrasianas." Walker (1972), p. 359.

36 Criticada agudamente en de Vroey (1999).

37 Por razones que se discuten en el próximo epígrafe.
} 
LW sobre la obra de Gossen (Walras, 1885). La idea central del trabajo de LW es la crítica del teorema de satisfacción máxima que, según él, en la formulación de Gossen, es más un deseo que un teorema, al plantearse el posible reparto de las dotaciones iniciales entre los individuos de forma que pueda alcanzarse un máximo del bienestar social sin restricción alguna sobre los precios de equilibrio. Pero Jaffé añade a esta interpretación defendible argumentos más que discutibles sobre el efecto de la censura política francesa en la obra de $\mathrm{LW}^{38}$, o especialmente confusos como, por ejemplo, que el interés de LW por la demanda de mercado en vez de la individual es una evidencia de su sesgo normativo ${ }^{39}$. E, incluso, posiciones erróneas, como que la introducción de bons en la teoría de la producción y su omisión en la del intercambio se debe a que el tiempo necesario para los ajustes en esta última es muy pequeño en relación al que se requiere en la primera. Sobre la base de esta abigarrada mezcla, Jaffé concluye que el tâtonnement en los EEPP es una "utopía" coherente con el esquema normativo de la obra.

Ciñéndonos al tema que nos ocupa, lo sorprendente es que la nueva interpretación del tâtonnement es más adecuada en varios aspectos que la sostenida en 1967, al señalar que se trata de un proceso de ajuste "atemporal, simultáneo y mecánico", introducido por razones expositivas y de análisis. En sus propias palabras:

\begin{abstract}
"Cuando se examina a la luz de "la historia completa de la economía de Walras" la versión formal del tâtonnement walrasiano se revela no como una pieza de dinámica formulada deficientemente, sino como un ejercicio intencionadamente estático de análisis de estabilidad, sujeto a las mismas cualificaciones que orientan su teoría formal del equilibrio general." (Jaffé, 1981, p. 333).
\end{abstract}

\title{
6. Conclusiones: a modo de reinterpretación
}

El conjunto de problemas debatidos puede sintetizarse en cuatro temas, de importancia desigual, en los que existen interpretaciones alternativas y, con frecuencia, incompatibles. Trataré de argumentar en cada uno de ellos cuál es, en mi opinión, la posición más defendible y coherente con la obra de LW.

38 Ciertamente LW no pudo hacer carrera académica en su Francia natal, pero la mayoría de su obra, tanto teórica como aplicada, la realizó siendo catedrático en Lausanne, donde siguió manteniendo una actividad publicista de signo progresista muy intensa sin cortapisas políticas.

39 Como señala acertadamente Walker (1996), la carta de Piccard sobre la técnica de maximización aplicada a la demanda individual [ $\mathrm{n}^{\circ}$ 211, n (4), vol. I, pp. 309-311, traducida en Segura (1987), pp. 106-109] desbarata esta interpretación. 


\subsection{El problema del intercambio a precios falsos y la (in)coherencia analítica del tâtonnement en los EEPP}

Este es el tema estrella, porque del mismo dependen casi todos los demás. Supongamos, en la interpretación más negativa para LW, que en 1874 no era consciente de que su tátonnement no excluía el comercio a precios falsos, en cuyo caso los precios finalmente alcanzados no podrían ser los mismos que los que solucionaban matemáticamente su sistema de ecuaciones de equilibrio del intercambio y la producción, y que fueron las críticas analizadas en el apartado 3.1 las que le abrieron los ojos. Esto implicaría, simplemente, que no es mérito de LW la modificación de su tâtonnement inicial, pero no habilita a sostener ni que en la versión modificada de la cuarta edición admitía el comercio a precios falsos, ni que su teoría se basa en un error conceptual.

No parece discutible que la introducción de bons en su modelo de producción tuvo como único objetivo eliminar el comercio fuera de equilibrio. El problema parece radicar en que no introdujo los bons también en su modelo de intercambio. Pero:

- ¿cómo no tener en cuenta que Walras (1880), describiendo la bolsa y el arbitraje, escribió que los agentes no operan hasta haberse determinado el precio al que la demanda y oferta efectiva son iguales?

- ¿cómo es posible que, si se percató del problema en el caso del modelo de producción, no lo hiciera en el caso del intercambio, donde formula el teorema de las redistribuciones equivalentes?

- ¿ ¿por qué si en ninguna edición de los EEPP se dice que existe intercambio a precios falsos y éste resulta incompatible con el teorema de las redistribuciones equivalentes es más razonable suponer que cometió una inconsistencia lógica grave en vez de interpretar que consideraba (implícitamente) que no existía intercambio a precios falsos?

- ¿cómo ignorar que Walras (1875 y EEPP) distinguía con nitidez entre eficiencia asignativa y optimalidad social, que consideraba que la justicia en el intercambio era incompatible con las redistribuciones y que el equilibrio competitivo era justo?

- ¿ ¿por qué suponer que LW no era consciente antes de 1900 de que si había comercio falso en la producción, los beneficios no podrían ser nulos y, por tanto, que LW no era consciente de la Ley de Walras?

Todas las interpretaciones son discutibles pero, en este caso, existiendo abundante evidencia de que LW no ignoraba que el intercambio a precios falsos planteaba problemas lógicos relevantes en su construcción teórica - e, incluso, de justicia - parece más razonable interpretar que no logró una formulación adecuada del tâtonnement - 
un problema complejo y original - hasta la cuarta edición de los EEPP (1900), que considerar que nunca fue consciente del verdadero problema y que vivió sumido en la inconsistencia lógica ${ }^{40}$.

\subsection{El tâtonnement como construcción abstracta o como representación de la realidad}

La opinión de que para LW el tâtonnement era una representación de cómo se ajustan los mercados en la realidad tiene una base aparentemente sólida: la machacona insistencia del autor en el paralelismo absoluto entre la forma de resolución matemática de las ecuaciones de equilibrio y la forma práctica en que el mercado alcanza el equilibrio, en que la solución teórica y empírica son "idénticas". Y también algunas interpretaciones límite, como la de Bortkiewicz de que el tâtonnement describe la senda que siguen los precios o la de Pareto de que representa la senda seguida por la mayor parte de los fenómenos económicos (Pareto, 1896-97, p. 25). Incluso las posiciones más matizadas de Patinkin (1965) y Jaffé (1967) —analizadas en los apartados 4.1 y 5 , respectivamente - defienden la interpretación 'realista' del tâtonnement.

Sin embargo, el caso más extremo de interpretación en términos de realismo es, como hemos visto, el de Walker, que proporciona una visión empirista de la obra de LW y, en particular, del tâtonnement. Walker parte de un a priori en su interpretación: considerar que los modelos sólo pueden interpretarse en términos de fenómenos observables (Lendjel, 1997) y puesto que el tâtonnement trata de ser "una descripción de los ajustes reales" (Walker, 1996, p. 51), el de la producción sin bons es más realista que con bons porque los mercados reales se encuentran en estado de permanente desequilibrio ${ }^{41}$.

Pero resulta difícil sostener esta visión del tâtonnement como una descripción real del funcionamiento de los mercados. En una intervención ante la Academia de Ciencias Morales y Políticas francesa de fecha tan temprana como 1873, explicando el objetivo de la teoría matemática del intercambio, LW señala:

"Lo que la teoría matemática del intercambio persigue es, exclusivamente [...] la expresión matemática de este mecanismo de la libre competencia. Sin duda, incluso en esta operación analítica esencialmente abstracta, general y teórica..." (Walras, 1874, p. 9).

40 Ver Rebeyrol (2002) para más argumentos en esta línea interpretativa en su contestación al trabajo de Bridel y Huck (2002b).

41 Esto, incluso, le permite ir más allá, y sostener que la segunda y tercera ediciones de los EEPP de la "fase madura" de LW en que la producción se encuentra "en continuo desequilibrio" son analíticamente superiores a la cuarta - correspondiente a la "fase final" de decadencia de LW-, en que nada hay en desequilibrio y el modelo de EGC es "fragmentario y defectuoso". 
Y en los EEPP indica con precisión que "para aproximarnos cada vez más a la realidad" hay que pasar de la hipótesis de un mercado anual periódico a uno permanente, éste sí en continuo desequilibrio en el que "no existe el día en que la oferta efectiva de servicios y de productos se iguala a la demanda efectiva y en que el precio de venta de los productos se iguala al coste de los servicios utilizados en su producción", en el que existe "desvío de servicios productivos desde las empresas con pérdidas a las empresas con beneficios", en el que el aumento de la producción no logra reducir el precio de venta superior al coste de producción... Pero aún así: "Más capaces seremos de controlar o prevenir estas crisis cuanto mejor conozcamos las condiciones ideales del equilibrio" (EEPP, Lección 35: 322, pp. 619-620).

Parece poco discutible que los sucesivos modelos de los EEPP perseguían determinar "condiciones ideales de equilibrio" y proporcionar un análisis "esencialmente abstracto, general y teórico" de una economía competitiva, y no describir cómo funcionaban los mercados reales. Por tanto, tiene poco sentido, tanto criticar los tâtonnements por su falta de realismo, como tratar de defenderlos frente a ataques que consideran no describen exactamente cómo realizan los ajustes de precios los mercados competitivos del mundo real. No era eso lo que perseguía LW y su insistencia en la identidad de las soluciones de mercado y matemática se puede interpretar con facilidad en términos de su afán por convencer de que la aplicación del método matemático a la economía traería consigo a ésta las mismas ventajas que, dos siglos antes, había supuesto para la física ${ }^{42}$. Hacía bien Jaffé en 1981 en dudar del 'aire de relevancia empírica' que atribuyó al tâtonnement en 1967.

Pero lo que resulta contradictorio de algunas interpretaciones es mantener la idea de que el tâtonnement para LW era una descripción de la realidad (un instrumento abstracto expositivo) y, simultáneamente, que excluyó (aceptó) los intercambios a precios de no equilibrio. Si era una descripción de la realidad, LW aceptaba que los mercados se encontraban en desequilibrio y, por tanto, se realizaba comercio a precios falsos. Si era un instrumento analítico expositivo, la coherencia interna del modelo de EGC exigiría evitar dicho comercio.

42 Por citar uno de los numerosos textos sobre el tema: “El siglo XX, que no se encuentra lejos, hará sentir la necesidad, incluso en Francia, de poner las ciencias sociales en manos de hombres de cultura amplia, acostumbrados a razonar tanto inductiva como deductivamente y que se encuentren familiarizados con el razonamiento y la experiencia práctica. Entonces la economía matemática adquirirá un rango parejo al de la astronomía y la mecánica matemáticas, y ese día se hará justicia a nuestro trabajo." EEPP, Prólogo a la $4^{\text {a }}$ edición definitiva (1900), p. 136. 


\subsection{El carácter estático o dinámico del tâtonnement}

El principal responsable de la interpretación dinámica del tâtonnement fue Edgeworth, pero en esta misma línea se encuentran las posiciones de Bortkiewicz, Pareto, Lange, Schumpeter, Patinkin o Negishi. No parece que esta interpretación tenga mucho fundamento. La cita fragmentaria del subepígrafe anterior (EEPP: Lección 35: 322, pp. 619-620) muestra con claridad que la dinámica para LW estaba vinculada a situaciones de desequilibrio en que, durante períodos apreciables de tiempo, no se cumplía la igualdad entre ofertas y demandas, los precios no se igualaban a los costes de producción unitarios y las variaciones de la producción no lograban conducir los precios en la dirección adecuada. Si el tâtonnement es un instrumento analítico abstracto que excluye los intercambios a precios falsos cuyo objetivo es explicar estilizadamente cómo pueden encontrarse los precios de equilibrio, nada tiene que ver con los procesos dinámicos que, precisamente, impiden que los tâtonnements se completen, porque alteran constantemente los datos del problema.

Otra cosa distinta es que el tâtonnement se haya vinculado, con fundamento, al análisis de la estabilidad del EGC. Es cierto que, como señaló agudamente Schumpeter (1971, p. 1096), el objetivo de LW era lo que en términos actuales llamaríamos resolver el problema de existencia ${ }^{43}$ — que el tâtonnement permitía obtener la solución del sistema de ecuaciones definitorias del EGC, el cual, por tanto, existía-. Pero al utilizar para ello un procedimiento que trata de demostrar que las fuerzas de la oferta y la demanda pueden terminar alcanzando los precios de equilibrio a partir de un vector inicial de precios cualquie$r a$ "voceado al azar" resulta inevitable vincular el tema a la estabilidad (global) del EGC.

Esto, sin embargo, no implica que el tâtonnement tenga un carácter dinámico, ni siquiera de dinámica implícita e imperfecta como pensaba Lange, porque la estabilidad que puede discutirse con el tâtonnement walrasiano ${ }^{44}$ es exclusivamente la que definió Hicks (1939) como estabilidad estática perfecta y no incluye formulación alguna de la dinámica temporal de los precios.

\subsection{La (in)corrección de la demostración de convergencia en los EEPP}

Como hemos visto, diversos autores han señalado que LW no demostró la convergencia de sus tâtonnements de forma satisfactoria. Se trata de una crítica irrelevante, semejante a la que señala que no demostró rigurosamente la existencia del EGC. No

43 Sobre la lectura del tâtonnement en términos de la solución de un sistema de ecuaciones o la descripción de un proceso en tiempo real es interesante Costa (2002).

44 Es decir, tal y como lo formuló Walras y no como categoría general (Negishi, 1962) o con la estructura dinámica explícita en Samuelson (1941). 
habría podido. La primera demostración rigurosa de existencia se debió a Wald (1933-35), exigiendo la aplicación de teoremas de punto fijo. La demostración rigurosa de las condiciones que garantizaban el tipo de tâtonnement definido por LW no vio luz hasta 1939, de la mano de Hicks, e implicaba propiedades de la matriz jacobiana de las funciones de exceso de demanda.

Cuando LW discute la posible multiplicidad de los equilibrios competitivos, la discusión para el caso de intercambio de dos bienes es correcta, pero elimina la posibilidad de equilibrios múltiples en el caso de varias mercancías con la simple afirmación de que es menos probable que se den equilibrios múltiples si hay muchos bienes. Parece sensato interpretar que LW, no pudiendo demostrar las condiciones de unicidad del equilibrio, evadió el problema con una afirmación gratuita, porque era consciente de que si hubiera soluciones múltiples no podría determinarse cuál sería el equilibrio del mercado y eso planteaba problemas en la arquitectura de sus EEPP.

Sin embargo, si nos situamos en 1874, no se podía argumentar la hipotética convergencia del tâtonnement de otra forma que la que utilizó LW. En lo esencial, el proceso walrasiano es ${ }^{45}$ :

1. Se vocea un vector de precios cualquiera p' para el que la función de exceso de demanda de $(B) z_{b}\left(p^{\prime}\right)$ es no nula.

2. Se obtiene un $\mathrm{p}^{\prime \prime}{ }_{\mathrm{b}}$ que hace $\mathrm{z}_{\mathrm{b}}\left(\mathrm{p}^{\prime \prime}{ }_{\mathrm{b}^{\prime}} \mathrm{p}^{\prime}\right)^{46}$ nula.

3. Se busca el $\mathrm{p}^{\prime \prime}{ }_{\mathrm{c}}$ que hace $\mathrm{z}_{\mathrm{c}}\left(\mathrm{p}^{\prime \prime}{ }_{\mathrm{b}^{\prime}} \mathrm{p}^{\prime \prime}{ }_{\mathrm{c}^{\prime}} \mathrm{p}^{\prime}\right)=0$ y así sucesivamente para todos los bienes.

Cuando se obtiene el vector de precios $\mathrm{p}^{\prime \prime}$ que hace nulo el exceso de demanda del último bien, es claro que $\mathrm{z}_{\mathrm{b}}\left(\mathrm{p}^{\prime \prime}\right)$ será no nula. $\mathrm{LW}$ considera que $\mathrm{z}_{\mathrm{b}}\left(\mathrm{p}^{\prime \prime}\right)$ se encuentra más cerca de cero que $\mathrm{z}_{\mathrm{b}}\left(\mathrm{p}^{\prime}\right)$, por lo que la repetición del proceso 1-3 terminará logrando el equilibrio. Las razones que aduce son que los cambios de $\mathrm{p}_{\mathrm{c}}{ }_{\mathrm{c}}$ a $\mathrm{p}^{\prime \prime}{ }_{\mathrm{c}^{\prime}} \mathrm{p}_{\mathrm{d}}{ }_{\mathrm{d}}$ a $\mathrm{p}^{\prime \prime}{ }_{\mathrm{d}} \ldots$ tienen sobre la demanda de (B):

- efectos indirectos,

- que se compensan hasta cierto punto entre sí.

Que son indirectos no ofrece duda; que se compensen parcialmente entre sí sucederá siempre que los bienes tengan con el (B) unas relaciones de sustituibilidad bruta y otros de complementariedad bruta. Ni lo uno ni lo otro aseguran la convergencia del proceso, pero tiene sentido señalar que la exigencia de que los efectos indirectos en su conjunto sean menos fuertes que el efecto directo de la variación del

45 Uzawa (1960) señaló con acierto que el tâtonnement se presenta, primero, como un proceso de ajuste de precios sucesivo (EEPP, Lección 12: 127-129) y se resume como un proceso simultáneo (EEPP: Lección 12: 130). Pero, aparte del tecnicismo del comentario, su demostración de estabilidad se hace bajo el supuesto de ajuste sucesivo, que es la versión más acorde al espíritu de los EEPP.

46 Notación: $z_{x}\left(p^{\prime \prime}{ }_{x^{\prime}} p^{\prime}\right)$ es el valor del exceso de demanda de $(X)$ para un precio de $(X)$ como $\mathrm{p}^{\prime \prime}{ }_{\mathrm{x}} \mathrm{y}$ los precios del vector $\mathrm{p}^{\prime}$ para los restantes bienes. 
propio precio es la condición moderna suficiente de estabilidad del tâtonnement dinámico ${ }^{47}$. Por lo tanto LW no demostró las condiciones de estabilidad estática, pero señaló el camino: una condición suficiente es que los efectos del propio precio sean más fuertes que la suma de los efectos precio cruzados ${ }^{48}$.

No parece poco si se tiene en cuenta que 108 años más tarde, en una revisión del estado de la investigación sobre la estabilidad del EGC, Hahn escribía:

“ [...] habremos de concluir que aun no disponemos de una teoría satisfactoria de la mano invisible [...]. Pero el tema [de la estabilidad del EGC] en su conjunto tiene un penoso aspecto ad hoc." (Hahn, 1982, pp.746-747).

\section{Bibliografía}

BERTRAND, Joseph (1996 [1883]): “Théorie mathématique de la richesse sociale, par Léon Walras, professeur d'économie politique à l'Académie de Lausanne, 1883. Recherches sur les principes mathématiques de la théorie de la richesse, par Augustin Cournot, Paris, 1838“, en BRIDEL, Pascal (ed.), Le chêne et l'architecte: un siècle de comptes rendus bibliographiques des Eléments d'économie politique pure de Léon Walras, París y Génova, Droz, pp. 195-202.

BORTKIEWICZ, Ladislaus von (1890): "Review of Eléments d'économie politique pure, ou Théorie de la richesse sociale, $2^{\text {nd }}$ ed., by Léon Walras", Revue d'Économie Politique, 4, pp. 80-86.

BRIDEL, Pascal y HUCK, Élisabeth (2002a): “Yet another look at Léon Walras's theory of tâtonnement", The European Journal of the History of Economic Thought, 9, pp. 513-540.

—(2002b): "Walras's tâtonnement: a reply to Rebeyrol and Costa", The European Journal of the History of Economic Thought, 9, pp. 559-567.

COSTA, Manuel Luis (2002): "Comment on 'Yet another look at Léon Walras's theory of tâtonnement'"', The European Journal of the History of Economic Thought, 9, pp. 550-558.

47 Que el jacobiano de las funciones $\mathrm{z}(\mathrm{p})$ tenga diagonal dominante negativa; que los elementos de la diagonal principal sean negativos y los restantes positivos (todos los bienes substitutos brutos, etc.). Véase Hahn (1982).

48 Frente a esto, el comentario formalmente correcto de Jaffé (1967) de que con las funciones de utilidad que utilizaba LW los bienes eran independientes, tiene un interés muy limitado y se asemeja bastante al tipo de críticas que él mismo calificaba irónicamente como "sutilezas del análisis moderno" de algunos economistas matemáticos. 
EDGEWORTH, Francis Ysidro (1881): Mathematical Psychics, Londres, Kegan Paul \& Co.

-(1889): “The Mathematical Theory of Political Economy, Eléments d'économie politique pure. Par Léon Walras", Nature, 40 (1.036, 5 sept.), pp. 434-436.

-(1891): "Théorie mathématique de l'offre et de la demande et le coût de production", Revue d'Économie Politique, 5, pp. 10-28.

-(1925): Papers Relating to Political Economy, 3 vols., Londres, Macmillan.

HAHN, Frank (1982): "Stability", en ARROW, Keneth e INTRILIGATOR, Michael (eds.), Handbook of Mathematical Economics, Amsterdam, North-Holland, vol. II, cap. 16, pp. 745-793.

HICKS, John R. (1939): Value and Capital, $2^{\mathrm{a}}$ edición (1946), Oxford, Oxford University Press.

HUCK, Élisabeth (2001): “La neutralité du tâtonnement au regard de la répartition de la richesse dans la théorie de la production de Léon Walras. Le cas du tâtonnement sans bons des trois premières éditions des Éléments d'économie politique pure», Revue Économique, 53, pp. 729-738.

JAFFÉ, William (1954): Elements of Pure Economics, Londres, Allen \& Unwin.

-(1965) (ed.): Correspondence of Léon Walras and Related Papers, 3 vols., Amsterdam, North-Holland.

—(1967): "Walras's Theory of Tâtonnement: A Critique of Recent Interpretations", Journal of Political Economy, 75, pp. 1-19.

-(1977): "The Normative Bias of the Walrasian Model: Walras versus Gossen", Quarterly Journal of Economics, 91, pp. 371-387.

—(1981): "Another Look at Léon Walras's Theory of Tâtonnement", History of Political Economy, 13, pp. 313-336.

KALDOR, Nicholas (1934): "A Classificatory Note on the Determinateness of Equilibrium", Review of Economic Studies, 1, pp. 122-136.

KUENNE, Robert E. (1961): “The Walrasian Theory of Money: An Interpretation and a Reconstruction", Metroeconomica, 13, pp. 94-105.

LANGE, Oskar (1936): "On the Economic Theory of Socialism", Review of Economic Studies, 4, pp. 53-71 y 123-142.

-(1944): Price Flexibility and Employment, Indiana, Bloomington.

LENDJEL, Emeric (1997): “Le 'biais empiriste' dans l'interprétation de Walker du tâtonnement walrassien", Économies et Sociétés, 26, pp. 47-84.

NEGISHI, Takashi (1962): “The Stability of a Competitive Economy: A Survey Article", Econometrica, 30, pp. 635-669.

PARETO, Vilfredo (1896-97): Cours d'économie politique, 2 vols., Lausanne, Rouge.

PATINKIN, Don (1965 [1956]): Money, Interest and Prices: An Integration of Monetary and Value Theory, New York, Harper \& Row.

REBEYROL, Antoine (2002): “'Yet another look?' A comment", The European Journal 
of the History of Economic Thought, 9, pp. 541-549.

SAMUELSON, Paul Anthony (1941): "The Stability of Equilibrium: Comparative Statics and Dynamics", Econometrica, 9, pp. 97-120.

SCHUMPETER, Joseph Allois (1971 [1954]): Historia del Análisis Económico, Barcelona, Ariel.

SEGURA, Julio (1970): “Una revisión del problema de existencia del equilibrio competitivo", Anales de Economía, $3^{\mathrm{a}}$ época, 8, pp. 225-268.

-(1987): Elementos de Economía Política Pura o Teoría de la Riqueza Social, edición traducida, anotada y comentada de Walras (1926) y Jaffé (1954), Madrid, Alianza Editorial.

SOLOW, Robert (1956): "Review of Elements of Pure Economics", Econometrica, 24, pp. 87-89.

STIGLER, George J. (1965): “Textual Exegesis as a Scientific Problem”, Economica, 32, pp. 447-450.

UZAWA, Hirozumi (1960): "Walras's Tâtonnement in the Theory of Exchange", Review of Economic Studies, 27, pp. 182-194.

VROEY, Michel de (1999): “Transforming Walras into a Marshallian Economist: A Critical Review of Donald Walker's Walras's Market Models", Journal of the History of Economic Thought, 21, pp. 413-435.

WALD, Abraham (1933-35): “Über die eindeutige positive Lösbarkeit der neuen Produktions gleichungen, I" y "Über die Produktiongleichungen der ökonomischen Wertlehre II", ambos en Ergebnisse eines Matematischen Kolloquiums (1933-34 y 1934-35). Traducción al inglés en BAUMOL, William J., y GOLDFIELD, Stephen (1968) (eds.), Precursors of Mathematical Economics: An Anthology, London School of Economics Series Reprint of Scarce Works on Political Economy, $\mathrm{n}^{\circ} 19$, pp. 281-293. Traducción al español en SEGURA, Julio y Rodríguez Braun, Carlos (1998) (eds.), La economía en sus textos, Madrid, Taurus, pp. 344-361).

WALKER, Donald A. (1972): "Competitive Tâtonnement Exchange Markets", Kyklos, 25, pp. 345-363.

—(1987a): "Walras's Theories of Tâtonnement”, Journal of Political Economy, 95, pp. 758-774.

—(1987b): "Edgeworth versus Walras in the Theory of Tâtonnement", Eastern Economic Journal, 13, pp. 155-165.

-(1996): Walras's Market Models, Cambridge, Cambridge University Press.

WALRAS, Léon (1874): "Principe d'une théorie mathématique de l'échange", Journal des Economistes, serie 3, 34 (100), pp. 5-21.

-(1874-77): Éléments d'Économie Politique Pure ou Théorie de la Richesse Sociale, Lausanne: Corbaz; $2^{\mathrm{a}}$ edición 1889, Lausanne: Rouge; $3^{\mathrm{a}}$ edición 1896, Lausanne: Rouge; $4^{a}$ edición 1900, Lausanne: Rouge; edición definitiva, 1926, Paris: Pichon et Durand-Auzias. 
—(1875): “L'Etat et les chemins de fer", Reoue du Droit Public et de la Science Politique, 7 (1897), pp. 417-36; 8 (1897), pp. 42-58 [incluido en WALRAS, Léon (1898), Etudes d'économie politique appliquée (Théorie de la production de la richesse social), Lausanne, F. Rouge; y París, F. Pichon, pp. 193-232].

-(1880): "La bourse, la spéculation et l'agiotage", en WALRAS, Léon (1898), Etudes d'économie politique appliquée (Théorie de la production de la richesse social), Lausanne, F. Rouge; y París, F. Pichon, pp. 401-445.

-(1885): "Un économiste inconnu: Hermann-Henri Gossen", Journal des économistes, 30, pp. 68-90.

-(1896): Etudes d'économie sociale (Théorie de la répartition de la richesse sociale), Lausanne, F. Rouge; y París, F. Pichon.

-(1898): Etudes d'économie politique appliquée (Théorie de la production de la richesse social), Lausanne, F. Rouge; y París, F. Pichon.

WICKSELL, Knut (1934): Lectures on Political Economy, vol. I, General Theory, Londres, Routledge \& Kegan Paul. 